\title{
EXPERIMENTAL VALIDATION OF FLEXIBILITY PROVISION BY HIGHLY DISTRIBUTED DEMAND PORTFOLIO
}

\author{
M. H. Syed and G. M. Burt \\ University of Strathclyde, Scotland \\ mazheruddin.syed@strath.ac.uk
}

\author{
R. D'Hulst and J. Verbeeck \\ EnergyVille-VITO, Belgium \\ reinhilde.dhulst@vito.be
}

\begin{abstract}
The wide scale deployment and utilization of demand side management for the provision of frequency balancing service provision is hindered by the lack of proof of performance evaluation of such mechanisms. In this paper, the pre-qualification testing approaches for performance evaluation of frequency balancing service provision by highly distributed demand portfolio are discussed. Preliminary experimental results and challenges that arise of the pre-qualification tests conducted on a highly distributed flexible resource portfolio being managed by a multi-agent based demand side management technology are presented and the need for expansion of present day testing procedures discussed.
\end{abstract}

\section{INTRODUCTION}

In any electrical power network around the world, the transmission and distribution system's integrity and stability is highly dependent upon adequate procurement and prompt delivery of ancillary services for which the system operators set out a large portion of their budget. For instance, the Transmission System Operator (TSO) of Great Britain, National Grid, paid a sum of over $£ 31.6$ million (48 million US dollars) during the month of August 2015 to ensure the availability and provision of ancillary services [1]. There is an increasing interest in the provision of frequency balancing services by demand side which is evident by the fact that the ancillary service provision by demand side has doubled in Great Britain in the past months compared to the year before. To aid and encourage demand side to participate in frequency balancing services, aggregation models are being proposed by system operators. Such a model enables the demand side aggregators to aggregate the minimum volume of reserves that are required for participation in frequency balancing services. The system operator will provide a list of approved aggregators for such a model that will bolster the confidence of demand side customers and encourage their participation.

Frequency balancing service provision is set to become more critical. The flexible devices that are present in the network today (for example heat pumps and combined heat and power units) and the flexible devices that are expected to be the major part of the future demand (electric vehicles), will play a major role in providing frequency balancing services in the near future. The provision of frequency balancing services by such diverse and highly distributed flexible resources has been demonstrated at field trials by Eco-Grid in Bornholm, NINES in Shetland, Low Carbon London in London, PowerMatching City in Hoogkerk and LINEAR in Flanders [2-6]. These field trials have contributed to the proof of concept of the ability of such diverse and highly distributed flexible resources to effectively participate in frequency balancing services. Before any service provider (a service provider here refers to aggregators of demand response and battery storage systems) can participate in frequency balancing service provision they are subject to pre-qualification tests. The pre-qualification tests are conducted to verify the service provider's conformance to the requirements of the frequency balancing service. Great Britain has led the way by defining the pre-qualification tests for aggregators of demand response and this is expected to be followed by other countries. However, the present day pre-qualification tests have not been designed to rigorously evaluate the ability of diverse and highly distributed flexible demand portfolios to conform to frequency balancing services.

In this paper, the limitations of present day prequalification are discussed. The need to expand these tests to accommodate a portfolio of diverse and highly distributed demand portfolio is presented. An experimental example will reveal some of the challenges faced when incorporating a multi-agent based demand side management technology managing a distributed flexible demand portfolio.

\section{FREQUENCY BALANCING SERVICES}

The types of frequency balancing services that a service provider can participate in can be broadly classified into two types: a) dynamic frequency balancing services and b) static frequency balancing services. Dynamic frequency balancing services can be defined as the services in which the service provider needs to adjust their response continuously depending upon the frequency of the network. This type of service is generally utilized for the purpose of frequency regulation. Static frequency balancing services can be defined as the services in which the service providers deliver a block response when requested and for a set duration of time. Their response is not adjusted based on the frequency. These services are designed by the system operators in order to achieve a precise coordinated frequency response which is essential for system security. These services vary largely from country to country which is understandable as the nature of the power system varies largely from country to country. The examples of such services in the Great Britain are Firm Frequency Response (dynamic) and Frequency Control by Demand Management (static).

The week ahead reserve procurement tender process in Great Britain limits the type of devices that can participate as is evident from the 22 demand side aggregators' preference for resources greater than $250 \mathrm{~kW}$ in size (large HVAC units in particular). Planning the participation of a portfolio of smaller flexible devices a week ahead in frequency balancing services poses a significant challenge. 
Realizing the potential of future flexible devices that will be present within a network, Belgian transmission system operator, ELIA has proposed a platform called "Bid Ladder" for frequency restoration that allows the flexibility to participate up to an hour ahead [8]. The proposed platform recognizes the various different response times of devices that might exist within the network and therefore also offers participation based on various speeds. This opens up the possibility for demand side participation and encourages their involvement. It should be noted that the bid ladder platform is not exclusive to demand side participants.

Bid Ladder falls under the category of static frequency balancing services. Each provider of flexibility needs to bid in, an hour ahead, the amount of reserves that it would be able to provide each quarter hour for the following hour. Based on all bids that are received, a merit order list is formulated and the provider whose flexibility is accepted is notified. The activation of the reserves is based on a signal sent by the system operator and for a fixed number of 15 minutes up to an hour $(15,30,45$ or 60 minutes).

\section{Pre-Qualification Tests}

Every system operator details the technical specifications for each service and develops pre-qualification tests to ensure service providers' ability to abide by the services' technical specifications. For the same purpose, National Grid has set out technical guidance and testing procedures for service providers of frequency balancing services in [7]. Each service provider, on the other hand, designs and tailors their portfolio controller in order to conform to the technical specifications of the service they are interested to participate in. As the emerging frequency balancing service, Bid Ladder, is presently a proposal, the prequalification tests for it have not yet been characterized, but the technical requirements for the service have been outlined in the proposal and are as follows:

1. Service provider bid should be based on physical regulation.

2. Once the service provider reaches the requested power level, it should be capable to maintain a stable power level.

3. After the delivery of the reserves for the duration requested, the service provider should go back to normal consumption within 15 minutes.

4. For fast activations, the service provider should be able to ramp to full offered capacity within 15 minutes.

Based on the technical specifications of the Bid Ladder platform presented above, and National Grid's technical guidance document, some of the common criterion of frequency balancing services around which the prequalification tests are designed can be been summarized as follows:

Trigger: The activation of frequency balancing service is based on a trigger received by the service provider. There are two types of triggers that can be used for service activation: a) a signal sent by the system operator requesting activation or $b$ ) a threshold on one or more of the network observables that can be locally measured by the service provider. In the latter case, the threshold is often pre-determined by the service provider in order to have a coordinated response and frequency regulation/restoration. Therefore, the ability of a service provider to respond to a trigger is tested.

Duration of Response: The duration for which a service provider has to maintain their response upon activation is set by the operator and is specific to the type of service. The ability of the service provider to sustain their response for the duration set out by the system operator upon being called for activation is part of the pre-qualification test.

Volume of Response: The volume of response a service provider has to provide upon being called for activation is set by the operator and is specific to the type of service. The two capabilities that the operator would want to test are 1) the provider's ability to deliver the requested volume of reserve upon being called for activation and 2) the provider's ability to change the volume of response upon request, either due to a signal being received by the system operator or based on a threshold, after being called for activation.

Speed of Response: The speed at which the service provider needs to respond upon being called for activation is determined by the system operator and the ability of the service provider to respond within the set time frame is tested.

\section{Limitations of Present Day Pre-Qualification}

Each system operator designs their own set of prequalification tests for each service around the criterion identified above. The pre-qualification tests for existing services, like those described in [7], are designed based on the present day portfolios of service providers that comprise larger resources $(250 \mathrm{~kW}$ or greater in size for Great Britain). This section discusses the limitations of such an approach when applied to service provider with a portfolio comprising highly diverse and distributed flexible resources.

The robustness of response of a service provider is certified by repeating the tests a number of times and ensuring that the response of the provider is as expected for each test. Diverse domestic flexible devices that are highly distributed within the network may have varying characteristics, one such being the seasonality of the devices that are utilized. The traditional one-off robustness test is challenged by this. There is a danger of the portfolio of flexible devices being "pre-charged" to pass the traditional robustness tests.

The operator conducts tests to evaluate the service provider's ability to sustain the response for the duration called upon, but nevertheless there will be an error 
associated with each service provider's portfolio. The error associated with each portfolio is an essential characteristic that when determined would help the operator achieve a better coordination of frequency response. Also, an important characteristic that has been associated with distributed flexible demand portfolios is the rebound effect - this is generally not pre-determined. Furthermore, the operator checks if the full capacity response by the service provider is achieved within the time frame set by them, but the time it takes for the service provider to reach the full capacity response from the time the activation signal is received may not be determined. This limits the effective coordination of frequency within the network.

In general, it can be said that the traditional prequalification tests, although up to a certain extent do verify conformity to technical specifications, might not be valid for a future highly diverse and distributed demand portfolio and do not extract the essential characteristics of such portfolios. Extracting the characteristics of a portfolio of reserves under a service provider would be beneficial to both the system operator and the service provider.

\section{EXAMPLE STUDY}

In this section, an example study is presented by means of conducting an experimental pre-qualification test on a simulated portfolio of distributed flexible devices being managed by a multi-agent based demand side management technology. A pre-qualification test is developed based on the technical specifications of the Bid Ladder platform. The infrastructure of the University of Strathclyde's Dynamic Power Systems Laboratory's incorporating real time power hardware-in-the-loop co-simulation [10], enables seamless integration and testing of demand side management technologies for the provision of ancillary services. The following section will describe in detail the multi-agent controller itself, the testing procedure and the experimental results.

\section{Service Provider:}

The multi-agent based demand side management technology is managing a portfolio of distributed flexible devices, i.e., electric vehicles and battery systems. The controller of the portfolio is tailored to meet the technical specifications of Bid Ladder platform. The hour ahead, quarter hourly, reserve capacity bids need to be formulated in a way such that their activation is always possible irrespective of the consumption of the portfolio. The reserve capacity that can be bid is obtained by means of aggregating the flexibility constraint information from individual devices in the portfolio [9]. No additional resource specific information is required for bid formulation. The real-time activation and control of the consumption is based market based multi-agent control system. For the example study a portfolio that comprises of 500 electric vehicles and 500 battery systems is considered.

\section{Testing Procedure:}

A simple test (as shown in Figure 1) has been developed in this paper based on the technical specification of Bid Ladder platform and knowledge gained from tests described in [7]. As described earlier, each service provider bids in the quarter hourly capacity it can provide an hour ahead. The reserve activation is every quarter hour only. This means that if an activation signal is sent anywhere between 12:00 to $12: 14$, the service provider is required to activate his reserves only at 12:15. If an activation signal is received during the time the response is being provided, the service provider is expected to continue its response for the next quarter hour. The volume of activation might vary depending upon whether the bid has been declared as divisible or indivisible. For this case, it is assumed that the bid is indivisible (which means the service provider has to be called in for full capacity that it had bid for). The service provider is called for response three times and the expected response of the service provider is shown in Figure 1.

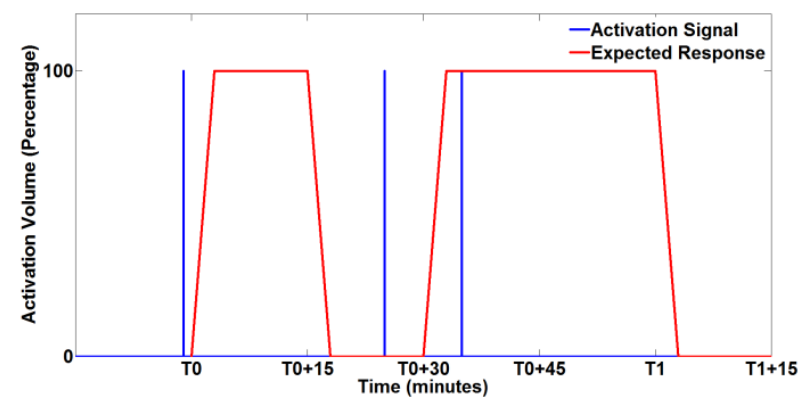

Figure 1: Pre-qualification tests and expected response

\section{Preliminary Results and Discussion:}

The quarter hourly capacity bid of the multi-agent based demand side management and its response to the test described in the earlier section are presented in Figure 2 and Figure 3 respectively. As the response provided by the service provider is by regulating the charge of the electric vehicles and the battery systems, the requirement of physical regulation is fulfilled. From the results presented in Figure 2, it is clearly demonstrated that the three other service specific requirements, namely, 1) maintaining stable power response throughout the duration for which the response has been called for, 2) ramping to full power capacity within 15 minutes of receiving the activation signal and 3) to return to normal consumption within 15 minutes after the duration for which the reserves had been requested, have been satisfied by the service provider. However it should be noted that although 1000 distributed devices are part of the service provider portfolio, only 718 participate during the three activations. One of the nontechnical requirements of the Bid Ladder platform states that only the resources that have participated in the prequalification process are allowed to participate in frequency balancing services. This renders about 282 resources unable to participate from within the portfolio of 


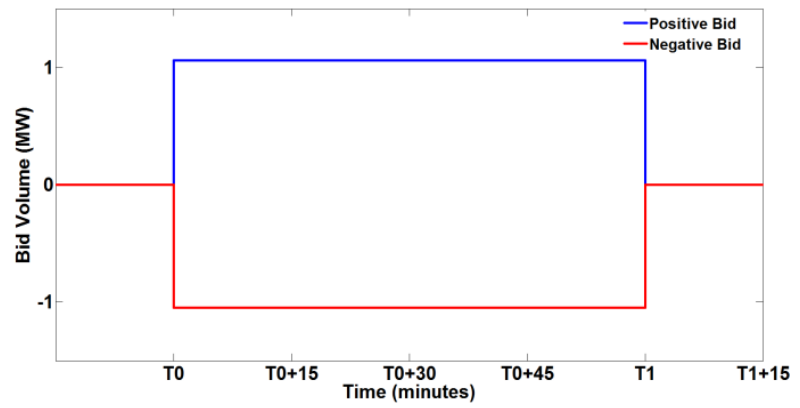

Figure 2: Quarter hourly capacity bid

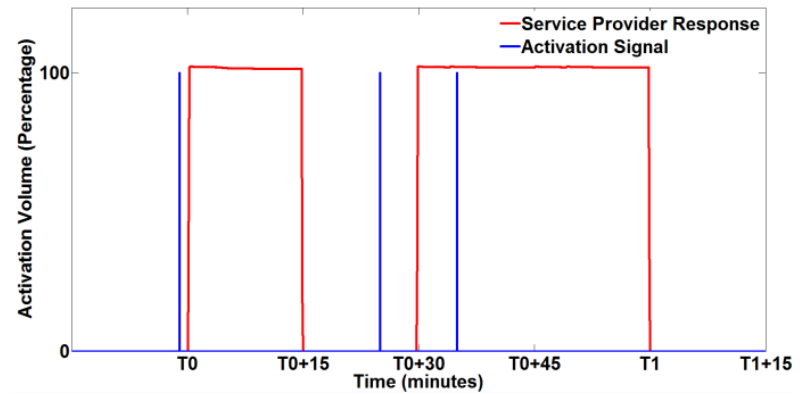

Figure 3: Service provider response

the service provider due to which the service provider might not be able to meet the requirement for minimum volume to bid into the service.

From the example study, it can be said that the present day tests that are mostly very specific to the type of service do not apply to portfolios with high penetration of distributed resources. There is a need for new tests to be developed that would evaluate the performance of diverse and highly distributed demand portfolios rigorously. In addition, extracting the essential characteristics, by means of developing new tests, on one hand will support the understanding of the system operator and enable accurate assessment and effective coordination of frequency response from flexibility available within the network. It will aid the system operator to design novel frequency balancing services based on the valuable information extracted. On the other hand, possessing the essential characteristics of its own portfolio will allow the service provider to assess their appropriateness for service provision. In the case of a new service has been developed and is being offered by the system operator, the results of these tests give a first-hand insight into whether the portfolio held by the service provider would be adequate or not. Further, it would also allow a room for further development of the portfolio of a service provider.

\section{CONCLUSIONS AND FUTURE WORK}

In this paper the pre-qualification test approaches to conform to the technical specifications of frequency balancing services have been discussed. Attention is drawn to the need of expansion of present day testing, that is specific to type of service, by highlighting the drawbacks of such an approach and presenting an example study that brings forth the additional value that the proposed expansion of tests add. Future work includes the identification and definition of characteristics of diverse and highly distributed portfolios within the network and development of tests and testing procedures that would extract the essential characteristics and rigorously ascertain the ability of such portfolios to participate in frequency balancing services.

\section{ACKNOWLEDGEMENT}

The work in this paper has been in part supported by the European Commission under the FP7 project ELECTRA (grant no: 609687) and in part by the Energy Technology Partnership. Any opinions, findings and conclusions or recommendations expressed in this material are those of the authors and do not necessarily reflect those of the European Commission.

\section{REFERENCES}

[1] "Monthly Balancing Services Summary, August 2015", [Online] Available: http://www2.nationalgr id.com/UK/Industryinformation/Electricity-transmission-operational-data/Report-explor er/Services-Reports/.

[2] L. Aleixo, A. Rosin, H. Saele, A. Z. Morch, O. S. Grande and I. Palu, 2013, "EcoGrid EU Project - Real Time Price Based Load Control and Economic Beneftis in a Wind Production Based System", Proceedings of $22^{\text {nd }}$ International Conference on Electricity Distribution, CIRED, Stockholm.

[3] M. Barnacle, S. Gill, M, J. Dolan and G. W. Ault, 2014, "A Multiobjective Assessment of the Future Potential of the Shetland Isles Active Network Management Scheme", CIRED Workshop, Rome.

[4] C. Marantes, R. Currie and D. Openshaw, 2011, "Low Carbon London- A Learning Journey", Proceedings of $21^{\text {st }}$ International Conference on Electricity Distribution, CIRED, Frankfurt.

[5] R. Kamphuis, B. Roossien, M. Eijgelaar, H. De Heer, J. van de Velde and A. Van Den Noort, 2013, "Real-Time trade dispatch of a Commercial VPP with Residential Customers in the PowerMatching City SmartGrid Living Lab", Proceedings of $22^{\text {nd }}$ International Conference on Electricity Distribution, CIRED, Stockholm.

[6] B. Dupont, P. Vingerhoets, P. Tant, K. Vanthournout, W. Cardinaels, T. De Rybel, E. Peeters and R. Belmans, 2012, "LINEAR Breakthrough Project: Large-Scale Implementation of Smart Grid Technologies in Distribution Grids", Proceedings of $3^{\text {rd }}$ IEEE PES International Conference and Exhibition on Innovative Smart Grid Technologies (ISGT), Berlin.

[7] N. Gargov and J. Sun, 2015, "Technical Guidance and Testing Procedure for Static and Dynamic Demand Response and Battery Storage Providers of Frequency Balancing Services", [Online] Available: http://www2.nationalgrid.com/UK/Services/Balanc ingServices/Frequency -response/Firm-Frequency-Response/FFRBridging/.

[8] "Proposal for the bidding \& activation process and balancing energy products for the bid ladder platform", [Online] Available: http://www.elia.be / /media/files/Elia/About-Elia/Users\%20Group/ Tas k-force-balancing/Design_Note_Bid_ladder_platfor m_v7External.pdf

[9] R. D'Hulst, J. Verbeeck, C. Caerts, M. H. Syed, A. Zaher and G. M. Burt, 2015, "Frequency restoration reserves: provision and activation using a multi-agent demand control system", Proceedings of 2015 International Symposium on Smart Electrical Distribution Systems and Technologies (EDST), Vienna.

[10] M. H. Syed, P. Crolla, G. M. Burt and J. K. Kok, 2015, “Ancillary service provision by demand side management: A real-time power hardware-in-the-loop co-simulation demonstration", Proceedings of 2015 International Symposium on Smart Electric Distribution Systems and Technologies (EDST), Vienna. 